Mémoires du livre

Studies in Book Culture

\title{
Maupassant, du livre au journal. Pour une matérialité de la littérature
}

Jérémy Naïm

Volume 8, numéro 2, printemps 2017

Le livre et le journal : croisements, prolongements et transformations The Book and the Periodical: Intersections, Extensions and

Transformations

URI : https://id.erudit.org/iderudit/1039700ar

DOI : https://doi.org/10.7202/1039700ar

Aller au sommaire du numéro

Éditeur(s)

Groupe de recherches et d'études sur le livre au Québec

ISSN

1920-602X (numérique)

Découvrir la revue

Citer cet article

Naïm, J. (2017). Maupassant, du livre au journal. Pour une matérialité de la littérature. Mémoires du livre / Studies in Book Culture, 8(2).

https://doi.org/10.7202/1039700ar

\section{Résumé de l'article}

Si deux textes disent littéralement la même chose mais qu'ils sont publiés sur deux supports différents, ont-ils encore le même sens? Cette vieille question que Borges avait posée à propos de Pierre Ménard, le présent article la repose à partir d'une nouvelle de Maupassant. On y verra entre autres choses que nos principaux outils d'analyse sont fondés sur une illusion rétrospective, qui, partant de ce que les oeuvres littéraires auraient toujours été pensées pour le livre, a donné à croire que celui-ci était un support neutre, sans contrainte ni influence.

Tous droits réservés ( C Groupe de recherches et d'études sur le livre au Québec, 2017 (c) (i) $\Theta$ 


\title{
MÉMOIRES DU LIVRE \\ STUDIES IN BOOK CULTURE
}

\section{MAUPASSANT, DU LIVRE AU JOURNAL. \\ Pour une matérialité de la littérature}

\author{
Jérémy NAÏM \\ Sorbonne Nouvelle / CRP19
}

Si deux textes disent littéralement la même chose mais qu'ils sont publiés sur deux supports différents, ont-ils encore le même sens? Cette vieille question que Borges avait posée à propos de Pierre Ménard, le présent article la repose à partir d'une nouvelle de Maupassant. On y verra entre autres choses que nos principaux outils d'analyse sont fondés sur une illusion rétrospective, qui, partant de ce que les œuvres littéraires auraient toujours été pensées pour le livre, a donné à croire que celui-ci était un support neutre, sans contrainte ni influence.

If two texts contain precisely the same words but are published in two different media, do they still have the same meaning? Borges posed this classic question concerning Pierre Menard. This article will reframe the question in terms of a short story by Maupassant. Among other topics, we shall see how our major analytical tools dwell on the retrospective delusion that a literary work had always been meant to be published in book form and that that book would be a neutral medium, without constraints or influences.

\section{Les formalismes, ou l'oubli du support}

Le support de publication a-t-il une incidence sur la forme d'une œuvre? Publier dans la presse ou en volume, dans un ouvrage illustré ou collectif, sont-ce des faits que le poéticien doit interroger? Ou bien doit-il se consacrer à l'analyse exclusive des faits de langue tels qu'accessibles dans la tissure des mots? 
Longtemps, les études formelles répondirent positivement à cette dernière question. Dans les années 1960, en plein linguistic turn, le structuralisme français engagea l'analyse littéraire dans une relation abstraite au langage. Furent rejetés hors de l'œuvre les faits sociaux et historiques, les éléments biographiques, ainsi que tout ce qui relève de la matérialité du livre, au profit de l'étude dématérialisée de leur surface verbale. C'était, de façon décisive, légitimer la recherche littéraire par une méthode d'apparence scientifique. On nomma "poétique » cette science des textes littéraires qui fournissait une série de concepts, l'« ellipse », le «narrateur", le «récit enchâssé » (toutes notions systématisées ou élaborées à cette époque-ci), permettant de dire à propos de l'œuvre « Comment est-ce que c'est fait ${ }^{1}$ ? ».

Nombreux furent ceux qui regrettèrent le déficit d'incarnation de ce nouveau formalisme. La sociologie de la littérature, en particulier, se développa dans l'interstice causé par la vague narratologique. Ainsi Robert Escarpit - pionnier en la matière -, Roger Chartier, Jean-Yves Mollier, firent mieux connaitre les conditions de production des œuvres, que ce soit en écrivant sur le monde des éditeurs, les structures de publication, ou en retraçant l'histoire du support « livre ». L’École de Constance s'intéressa à la réception des œuvres et ouvrit la voie à une sociologie de la lecture. Pierre Bourdieu, dans les Règles de l'art, appliqua à la littérature les concepts forgés tout au long d'une vie de sociologue ${ }^{2}$. Même Gérard Genette infléchit le formalisme excessif des années structuralistes en s'intéressant aux «seuils » dans un livre du même nom, c'est-à-dire à la signifiance de tout ce qui accompagne le texte - préface, épigraphe, notes, etc. Mais parce que ce dernier ignore les supports alternatifs au livre, son ouvrage fait apparaittre en négatif la difficulté des formalismes à penser le support dans l'histoire. Jusqu'à très récemment, et en dépit des avancées de la sociologie de la littérature, la prise en compte du support dans la construction formelle de l'œuvre a été négligée. Ce sont les travaux d'Alain Vaillant et de Marie-Ève Thérenty sur les rapports de la littérature et de la presse qui ont contribué à décloisonner ces deux lieux de pensée, le premier par son appel à prendre en considération la «communication littéraire ${ }^{3}$ » dans l'analyse des formes, la seconde, par son manifeste pour une « poétique historique du support ${ }^{4}$ ».

Que ces initiatives soient le fait de spécialistes de la presse, et du XIX ${ }^{e}$ siècle en particulier, n'est pas un hasard : le XIXe fut une période de double régime, 
où livre et journal servaient tour à tour de lieu de publication pour une même œuvre, avec, nécessairement, des transformations liées au passage d'un support à l'autre. Cette période est aussi celle où le livre devenait, à la faveur de l'amélioration des techniques d'impression et de l'entrée dans l'ère de masse, le support légitimant de l'œuvre. Or, ce que fait apparaitre ce double régime est l'incidence du support sur la forme des œuvres. La moindre correspondance d'écrivain le donne à voir dans les récriminations formulées, ici contre les prescriptions absurdes des libraires, ou là, contre les contraintes de la publication en colonne. Les écrivains ont toujours pris en compte le support pour lequel ils écrivaient. L'autonomisation de la littérature, qui, à la fin du siècle, confère au livre une aura particulière, reposait sur sa valorisation contre le journal. Ainsi du Livre de Mallarmé, qui était d'abord une réaction à «l'universel reportage » recommencé chaque jour dans la presse. En privilégiant l'atemporel sur l'éphémère, la stabilité sur l'instabilité, Mallarmé reconnaissait, au moins intuitivement, le rôle du support dans l'écriture littéraire.

À partir d'une nouvelle de Maupassant publiée dans la presse avant d'être éditée en volume, j'aimerais, à mon tour, évaluer l'incidence formelle que le support de publication, livre ou journal, peut avoir sur le sens d'une pratique. Je m'efforcerai de comprendre quelles sont les transformations impliquées par le passage du support-livre au support-journal, pour en interroger les conséquences sur l'héritage de nos catégories formelles.

\section{Maupassant dans le livre : « La Bécasse » (1883)}

Pourquoi Maupassant? Pour trois raisons. D’abord, parce qu'il appartenait à cette génération qui valorisa le livre contre le journal, et qu'il chercha, toute sa vie, à échapper aux contraintes et à la fatigue que lui imposait la publication hebdomadaire dans la presse. Ensuite, parce qu'il vécut toute sa carrière sous ce double régime, passant du journal au livre, se pliant aux exigences de l'un et de l'autre support. L'ensemble de ses publications révèlent une conscience en acte des supports d'écriture. Enfin, parce que la nouvelle dont je veux traiter connut, et toutes ne subirent pas le même sort, une transformation tout à fait singulière lors de son passage en livre, transformation qui tient, justement, aux particularités de ce support. 
En 1883, chez Rouveyre et Blond, éditeurs parisiens, paraissent les Contes de la bécasse. Le titre du recueil tire son nom du premier conte, intitulé « $\mathrm{La}$ Bécasse ». Il y est question du baron des Ravots, chasseur aguerri mais vieilli, empêché depuis cinq ou six ans par une paralysie de la jambe. Entre autres amusements d'une vie désormais sédentaire, le baron des Ravots a instauré dans sa maison la coutume du "conte de la Bécasse», dont la nouvelle donne l'explication suivante :

Au moment du passage de cette reine des gibiers, la même cérémonie recommençait à chaque dîner.

Comme ils adoraient l'incomparable oiseau, on en mangeait tous les soirs un par convive; mais on avait soin de laisser dans un plat toutes les têtes.

Alors le baron, officiant comme un évêque, se faisait apporter sur une assiette un peu de graisse, oignait avec soin les têtes précieuses en les tenant par le bout de la mince aiguille qui leur sert $\mathrm{de}^{5}$ bec. Une chandelle allumée était posée près de lui, et tout le monde se taisait, dans l'anxiété de l'attente.

Puis il saisissait un des crânes ainsi préparés, le fixait sur une épingle, piquait l'épingle sur un bouchon, maintenait le tout en équilibre au moyen de petits bâtons croisés comme des balanciers, et plantait délicatement cet appareil sur un goulot de bouteille en manière de tourniquet.

Tous les convives comptaient ensemble, d'une voix forte :

- Une, - deux, - trois.

Et le baron, d'un coup de doigt, faisait vivement pivoter ce joujou.

Celui des invités que désignait, en s'arrêtant, le long bec pointu devenait maittre de toutes les têtes, régal exquis qui faisait loucher ses voisins.

Il les prenait une à une et les faisait griller sur la chandelle. La graisse crépitait, la peau rissolée fumait, et l'élu du hasard croquait le crâne suiffé en le tenant par le nez et en poussant des exclamations de plaisir.

Et chaque fois les dîneurs, levant leurs verres, buvaient à sa santé.

Puis, quand il avait achevé le dernier, il devait sur l'ordre $\mathrm{du}$ baron, conter une histoire pour indemniser les déshérités.

Voici quelques-uns de ces récits ${ }^{6}$ : 
La nouvelle s'arrête sur le mot « récits » et les deux points qui le suivent. Elle se termine ainsi sur la page de gauche. Sur celle de droite, le titre du prochain conte est annoncé.

Ce premier texte a un statut à part dans le recueil. Matériellement, il se distingue. $\mathrm{Si}$, comme tous les autres, il commence en page impaire, après une page-titre, il est, en revanche, plus aéré. Il s'ouvre sur une demi-page blanche, alors que les autres contes voient leur titre répété et souligné en page d'ouverture; et il se ferme sur un même espace vide, et non sur le trait de clôture qu'on retrouve ailleurs. Même l'en-tête témoigne de cette différence : au lieu de l'alternance entre le titre du recueil (page paire) et celui de la nouvelle (page impaire), «La Bécasse» n'est surmontée que du seul Contes de la Bécasse.

Cette distinction physique de la nouvelle s'adapte à ses particularités de contenu. «La Bécasse » ne constitue pas une nouvelle à proprement parler, dans la mesure où elle ne raconte pas d'histoire. Elle ne contient ni fin véritable, ni élément déclencheur, et se trouve entièrement rédigée à l'imparfait ou au plus-que-parfait - s'il est possible d'écrire un récit au seul passé simple, la chose se complique avec l'imparfait, qui confine souvent à la description, statique ou d'action. Achevée sur deux points, la nouvelle joue un rôle d'encadrement pour toutes les autres; elle forme un dispositif de rassemblement à l'usage d'une collection de nouvelles. Le lecteur d'aujourd'hui qui connait ses formalismes pensera d'emblée au Décaméron ou à L'Heptaméron, ces grands recueils de la Renaissance construits selon un dispositif similaire. La narratologie parle d'« enchâssement narratif » (ou de « récit enchâssé ») pour évoquer le procédé qui consiste à faire raconter une histoire par un personnage, non pour le lecteur directement, mais à destination d'un autre personnage. Outre les recueils de la Renaissance que je viens de nommer, sont généralement rattachées à ce procédé Les Mille et Une Nuits - entièrement racontées par Shéhérazade au roi, son mari -, certaines séquences de La Princesse de Clèves - où la mère de la princesse, entre autres, lui rapporte des histoires de cour -, et quelques nouvelles de Barbey (les six Diaboliques), de Balzac (Sarrasine par exemple) et, bien entendu, de Maupassant.

Je me permets ici une digression qui aura son importance plus loin. Le récit enchâssé fait partie de ces catégories inventées par le formalisme, sur le 
fondement d'une attention exclusive aux relations verbales. La notion apparait au début du Xxe siècle, et le mot même d'« enchâssement» relève d'un emprunt à la linguistique générative. Avec cet outil, les structuralistes pensaient analyser une sorte de structure syntaxique du texte ${ }^{7}$, et particulièrement des recueils de nouvelles - abstraction faite de leurs contextes d'apparition. Au XIX ${ }^{e}$ siècle, en revanche, la perception des recueils tient davantage compte de leurs usages éditoriaux. Rien ne le montre mieux que la manière dont Henry Weber, éditeur anglais, envisage Les Mille et Une Nuits. Il en parle comme d'une "réserve [storehouse] d'inventions ingénieuses et d'images splendides ${ }^{8}$ » - le mot «réserve » signalant la représentation du recueil en tant que «collection », réunie dans un même volume sans autre intention que la diversification. Là où notre regard contemporain reconnait dans La Princesse de Clèves et Les Mille et Une Nuits la même situation narrative, le XIX ${ }^{e}$ siècle se représente (au moins) deux pratiques distinctes : celle du roman, où le récit de personnage imite plus ou moins l'écriture théâtrale (comme certains romans du XIXe siècle, notamment les romans américains, transposent à la littérature des techniques empruntées au cinéma); et celle du recueil, où le récit de personnage légitime le rassemblement d'histoires différentes au sein d'un même volume. Le «cadre » du recueil, comme on appelle parfois l'histoire qui précède et justifie le recueil, est une invention destinée à justifier une pratique associée au livre, sans lien avec les structures narratives. Et il n'y eut de comparaison entre recueil et roman que lorsqu'on ne perçut plus le geste éditorial à l'origine de la collection de nouvelles.

Si l'on revient aux Contes de la bécasse, on comprend alors deux choses. D'abord, que «La Bécasse », tout en imitant le Décaméron ou L'Heptaméron, n'a pas besoin d'ouvrir à des représentations de parole. De fait, aucune des nouvelles qui la suivent - à l'exception d'une seule, « La Folle », sur laquelle je vais revenir -, n'est rattachée à la cérémonie de la bécasse. Certaines sont racontées par des personnages, d'autres non; et quand elles le sont, c'est dans un lieu autre que le salon du baron des Ravots. Cette absence de la parole des personnages tient donc, et c'est le deuxième point, à la fonction très spécifique de "La Bécasse », qui consiste à justifier une collection hétérogène. La nouvelle liminaire remplace une préface potentielle. Elle est, au sens strict, purement livresque, en ceci qu'elle est pensée comme un discours spécifique au support-livre, dont l'initiative revenait peut-être autant à Maupassant qu'à ses éditeurs. On sait que, pour obtenir un volume 
qui ne fût pas trop mince, la publication de récits courts au XIXe siècle se faisait surtout par anthologie. Le rôle de «La Bécasse» était donc d'organiser une matière qu'on ne pouvait trouver que dans le livre, parce que spécifique à ce support. Or, il s'avère que «La Bécasse » a connu une autre vie avant d'ouvrir les Contes, et que sa genèse apporte une confirmation décisive à cette analyse 9 .

\section{Maupassant dans la presse : « La Folle » (1882)}

Maupassant, je l'ai rappelé en début d'article, pratiquait systématiquement la double publication en journal puis en livre. «La Bécasse » avait donc déjà été publiée, quelques mois auparavant, dans le numéro du Gaulois daté du 5 décembre 1882. Seulement, elle portait un autre titre, titre que j’ai mentionné à la section précédente : «La Folle ». «La Folle » de 1882 était en fait constituée de «La Bécasse », suivie de «La Folle » de 1883. La nouvelle racontait en continu la cérémonie des bécasses et le récit de l'un de ses vainqueurs. Au lieu de "Voici quelques-uns de ces récits», on lisait «Et voici ce que raconta M. Mathieu d'Endolin », personnage dont on ne savait rien de plus que ce nom donné à ce seul endroit du texte. Après cette phrase, suivaient trois astérisques, puis le texte correspondant à «La Folle » de 1883.

Un réflexe contemporain verrait dans cette version en journal un autre "récit enchâssé ", mais compte tenu de ce que l'on sait de l'histoire du procédé, cela serait difficile à défendre. Quand bien même les deux textes disent, littéralement, la même chose, le dispositif de «La Bécasse » et celui de «La Folle» de 1882 ne sont pas superposables. Leur support leur donne une fonction et un sens différents. Mais quelle était la fonction de ce que nous appelons « récit enchâssé » dans cette première version de « La Folle »?

Pour le comprendre, il faut penser la nouvelle dans son environnement originel, en lien direct avec son support. « La Folle » fut publiée en première page du Gaulois, quotidien plutôt mondain, à 15 centimes le numéro. Par comparaison, Le Petit Journal, qui avait démocratisé en 1863 l'accès à la presse, se vendait trois fois moins cher, soit à cinq centimes. Le public visé différait donc, mais le modèle d'écriture était proche. On ne composait pas de Une alors, mais des pages en continu, par adjonction de colonnes. En premier article de la première colonne, on plaçait généralement la 
« chronique », genre assez libre où le journaliste, s'adressant directement au lecteur, commentait une actualité, répondait à une polémique, enterrait quelque mort célèbre, ou simplement, racontait une histoire. Généralement, une périodicité renouvelait les chroniques, chaque jour de la semaine étant celui d'un chroniqueur, et donc d'un type de texte, différent. Même si les chroniques à la troisième personne se multipliaient, le dialogue avec le lecteur restait une règle tacite.

«La Folle » occupait donc la place dévolue d'ordinaire à la chronique. Elle constituait le premier article du numéro du cinq décembre, remplissant l'équivalent de deux colonnes, presque en pleine page - "presque", puisqu'un huitième de la page était meublé par l'ours et par le titre du journal. C'était une place habituelle pour Maupassant dans Le Gaulois. Le directeur de publication mettait là en avant une signature appréciée. Il ne fait pas de doute qu'en lisant «La Folle», les lecteurs reconnaissaient d'emblée un article à la dimension "littéraire », ou plutôt, et ici le choix des mots a son importance, à la dimension «narrative ». La question de la littérarité, en effet, était encore anachronique, et le plus souvent indécidable. Une semaine auparavant, avait paru, par exemple, une chronique d'Albert Delpit sous la

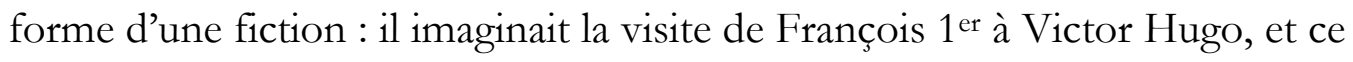
que le grand roi eût pu dire au grand poète ${ }^{10}$. La chronique était à la troisième personne, très écrite, recourant à une figure traditionnellement reconnue par la rhétorique, la prosopopée; mais dans le même temps, elle servait de discours biaisé pour parler du patriotisme et de la poésie. Elle ne répondait pas directement à un sujet d'actualité, mais s'appropriait des débats d'époque. Difficile a posteriori de savoir quel était son statut exact pour un lecteur de 1882. Il faut se contenter de remarquer, comme le fait Marie-Ève Thérenty, qu'il existait à l'époque un dialogue continu entre littérature et presse ${ }^{11}$, et parfois tel, qu'il est impossible de démêler ce qui relève de l'un ou de l'autre ${ }^{12}$.

«La Folle » constituait donc une chronique narrative, et en cela, respectait la règle tacite du dialogue avec le lecteur. Non un dialogue direct, puisque la nouvelle était à la troisième personne, mais un dialogue indirect, qui tenait au choix d'une narration contée. J'entends par là qu'au-delà des questions de personne (au sens grammatical), le récit était envisagé comme interlocution, avec les spécificités que cela suppose. L’une de ces spécificités reposait sur le fait que le récit ne commençait pas tout de go, mais succédait à un temps de 
prise de contact avec le lecteur, que Jakobson aurait nommé «phatique ». Outre la copie qu'il faisait gagner, ce temps permettait d'augmenter l'attente d'un rendez-vous déjà très attendu par le lecteur. Maupassant l'occupait en circonstanciant au maximum l'origine du récit. Cela collait au protocole d'authenticité relatif au journal. Jusqu'au milieu de l'année 1882, Maupassant, d'ailleurs, quand l'occasion se prêtait de contextualiser son récit, le faisait toujours à la première personne, en commentaire d'une actualité. Là, dans « Histoire corse » par exemple :

Deux gendarmes auraient été assassinés ces jours derniers pendant qu'ils conduisaient un prisonnier corse de Corte à Ajaccio. Or, chaque année, sur cette terre classique du banditisme, nous avons des gendarmes éventrés par les sauvages paysans de cette île, réfugiés dans la montagne à la suite de quelque vendetta. Le légendaire maquis cache en ce moment, d'après l'appréciation de MM. les magistrats eux-mêmes, cent cinquante à deux cents vagabonds de cette nature qui vivent sur les sommets, dans les roches et les broussailles, nourris par la population, grâce à la terreur qu'ils inspirent.

Je ne parlerai point des frères Bellacoscia dont la situation de bandits est presque officielle et qui occupent le Monte d'Oro, aux portes d'Ajaccio, sous le nez de l'autorité. La Corse est un département français; cela se passe donc en pleine patrie; et personne ne s'inquiète de ce défi jeté à la justice. Mais comme on a diversement envisagé les incursions de quelques bandits kroumirs, peuplade errante et barbare, sur la frontière presque indéterminée de nos possessions africaines!

Et voici qu'à propos de ce meurtre le souvenir me revient d'un voyage en cette île magnifique et d'une simple, toute simple, mais bien caractéristique aventure, où j’ai saisi l'esprit même de cette race acharnée à la vengeance ${ }^{13}$.

Ou ici, dans « Histoire d'un chien» :

Toute la presse a répondu dernièrement à l'appel de la Société protectrice des animaux, qui veut fonder un asile pour les bêtes. Ce serait là une espèce d'hospice, et un refuge où les pauvres chiens sans maitre trouveraient la nourriture et l'abri, au lieu du nœud coulant que leur réserve l'administration.

Les journaux, à ce propos, ont rappelé la fidélité des bêtes, leur intelligence, leur dévouement. Ils ont cité des 
traits de sagacité étonnante. Je veux à mon tour raconter l'histoire d'un chien perdu, mais d'un chien du commun, laid, d'allure vulgaire. Cette histoire, toute simple, est vraie de tout point ${ }^{14}$.

À partir de «Magnétisme », chronique publiée dans Gil Blas le 5 avril 1882, Maupassant expérimentait un récit circonstancié à la troisième personne, selon une scénographie qu'il reprendra à de très nombreuses reprises :

C'était à la fin d'un dîner d'hommes à l'heure des interminables cigares et des incessants petits verres, dans la fumée et l'engourdissement chaud des digestions, dans le léger trouble des têtes après tant de viandes et de liqueurs absorbées et mêlées.

On vint à parler du magnétisme, des tours de Donato et des expériences du docteur Charcot. Soudain ces hommes sceptiques, aimables, indifférents à toute religion, se mirent à raconter des faits étranges, des histoires incroyables mais arrivées, affirmaient-ils, retombant brusquement en des croyances superstitieuses, se cramponnant à ce dernier reste de merveilleux, devenus dévots à ce mystère du magnétisme, le défendant au nom de la science ${ }^{15}$.

Suivaient deux courts récits qui constituaient le cœur même de la nouvelle. La disparition du débat d'actualité répondait sans doute à une volonté de se détacher de l'écriture de presse - préoccupation qui revient régulièrement dans la correspondance de Maupassant -, sans toutefois remettre en cause son caractère conté, et donc la préparation phatique du récit. Les contemporains avaient-ils conscience de cette spécificité médiatique? Difficile à dire, mais l'on sait que Brunetière jugeait très défavorablement les nouvelles de Maupassant qui en prenaient le plus visiblement la forme :

[L]es nouvelles de M. de Maupassant s'encadrent, pour ainsi dire, comme des tableaux dans leur bordure, et nous donnent cette sensation du définitif et de l'achevé qui est le triomphe de l'art de composer. Je voudrais seulement qu'il usât un peu moins du procédé facile dont l'auteur de Carmen avait abusé avant lui. Cela consiste, on le sait, à introduire le récit principal d'une aventure de voyage ou de chasse, entre le commencement et la fin desquelles il s'encadre alors si naturellement... que l'artifice en saute aux yeux ${ }^{16}$. 
«Une aventure de voyage ou de chasse »... On retrouve enfin «La Folle». La particularité de cette nouvelle, comme le déplorait Brunetière, consistait à retarder le récit principal (celui de Mathieu d'Endolin) par un propos liminaire décrivant un repas de chasseur. Il ne faut pas se laisser prendre au mot «cadre » utilisé par Brunetière, qui n'est qu'un homonyme de celui qu'on utilise parfois pour désigner l'avant-propos du Décaméron. Le «cadre » de Brunetière ne désignait rien moins que le dispositif d'enchâssement, puisque, pour lui, tout récit était encadré, au sens de "composé ». Le défaut des nouvelles de voyage ou de chasse de Maupassant reposait donc sur un mauvais encadrement, c'est-à-dire sur une composition trop visible. Avec ce jugement, Brunetière opérait une forme d'analyse a posteriori, détachant les nouvelles de leur contexte d'énonciation pour ne s'intéresser qu'à leur caractère littéraire, et non plus à leur usage (à savoir, celui de nouvelles pour la presse). Brunetière jugeait le livre dans la nouvelle du journal, oubliant (ou feignant d'oublier, car il était, comme nombre de ses contemporains, très conscient des tropismes de l'écriture de presse) de signaler ce que le support devait à la forme de l'œuvre étudiée.

\section{L'illusion rétrospective}

Mais il va de soi, comme on l'a vu en relisant «La Bécasse », que le livre n'a rien d'un support neutre, qu'il donne des sens différents à des textes pourtant littéralement identiques. Il n'est pas impossible d'ailleurs que, dans l'histoire de l'enchâssement, "La Bécasse » représente un moment important. La transformation d'une nouvelle en cadre de recueil constitue un hapax de la littérature enchâssée, et si elle ne dit rien d'une notion partagée par le recueil et la fiction individuelle, elle traduit un possible, la trace d'un commun envisageable à deux types d'œuvres fonctionnellement différentes. Dans ce rapprochement, l'affaiblissement de l'usage d'origine exprime en réalité celui du support propre des œuvres et leur partage nouveau d'une matérialité commune. Sans doute Maupassant et ses contemporains n'ont-ils pas eu conscience de ces changements qui s'effectuaient en profondeur, selon un mouvement de fond dont il était difficile de percevoir les remous. Il restait bien dans «La Bécasse » et dans «La Folle» deux supports et deux rationalités différentes de ce que nous appelons a posteriori le récit enchâssé. Mais, en même temps, leur réversibilité 
signalait que des valeurs similaires commençaient à s'imposer à tous les textes, dont on ne neutralisait pas encore la vie éditoriale.

L'existence de ces deux supports suggère ainsi que le livre, loin d'être neutre, imposait des contraintes et entraînait une certaine forme. En reprenant la comparaison des nouvelles, on peut tirer deux caractéristiques majeures du support-livre.

D'abord, la fin du contact avec le lecteur. Le journal s'oblige à ce contact, parce que, plus que le livre, il dépend des réactions de ses lecteurs, que ce soit par des comportements d'achat ou par les lettres envoyées à la rédaction. Le lien du livre au lecteur, à l'inverse, est infiniment décalé dans le temps et ne justifie plus un dialogue continu. On ne mesure pas encore toutes les conséquences de cette transformation, qui marque, à la fois, l'évolution vers le roman à la troisième personne ${ }^{17}$ et la marginalisation d'un modèle de récit conté, comme on en trouve dans «La Folle » de 1882.

Corollaire inévitable de ce premier aspect, le livre atténue la fonctionnalité des énoncés écrits et transmet un énoncé décontextualisé. Dans le journal, la lecture de l'article obéit à une notion de consommation, rattachée à une actualité. L'article, de surcroît, est accompagné d'autres articles, qui le complètent ou le sectorisent. Mais sitôt lu, il est généralement oublié. Le livre, lui, stabilise l'écrit et le conserve plus longtemps. Or, pour ce faire, il tend à effacer tout contexte d'écriture. Ainsi, Brunetière ne jugeait-il pas des récits de presse, mais des chroniques publiées en livre, avec la distance sur leurs fonctions que cela supposait.

Ces deux traits, qui ne représentent sans doute pas les seules différences que l'on pourrait lister, n'impliquent aucun déterminisme. Ils disent seulement les usages qui ont été faits d'un support, mais d'autres usages du livre ou du journal demeurent envisageables. La généralisation du livre au $\mathrm{XX}^{\mathrm{e}}$ siècle verra d'ailleurs renaitre certains aspects liés au journal du XIXe siècle. Les conclusions que je tire sont plutôt de l'ordre du constat historique que de la causalité : la généralisation du livre dans la diffusion de l'œuvre littéraire a agi, dans la pratique, puis dans les discours sur cette pratique, comme l'effacement de tout rapport spatial (pas ou peu de contact avec le lecteur) ou temporel (décontextualisation) de l'énoncé littéraire. Soit, en définitive, la neutralisation de tout support. L'écrivain écrivant pour le livre pensait 
d'abord le Livre comme un objet hors du temps; et le critique considérait les livres du passé, en dehors de tout geste philologique, comme des œuvres décontextualisées. Je trace bien entendu un schéma caricatural, aux lignes beaucoup trop strictes, mais qui permet de comprendre l'émergence des formalismes au $\mathrm{XX}^{\mathrm{e}}$ siècle. Si l'on ne pense plus l'œuvre que comme livre, et ce livre, comme une abstraction, comment décrire une nouvelle comme « La Folle »? Le retard du récit, caractéristique d'une certaine écriture de presse, disparaissait inévitablement. Restait alors à inventer de nouvelles perspectives sur ce support abstrait qui fait passer le texte à travers l'espace et les âges. Ces nouvelles perspectives - dont le récit enchâssé est un exemple - reposent en principe sur une illusion rétrospective : qu'il existe quelque chose comme un langage commun aux œuvres, des catégories transhistoriques. Dans l'usage de nos outils d'analyse, nous sommes encore victimes de cette illusion. Elle nous oblige à penser la forme des œuvres, non seulement hors de tout espace-temps, mais en retrait de leurs différences matérielles. Un historien de l'art oserait-il comparer le dessin d'une poterie romaine à celui d'un tableau de Delacroix? Certainement pas. Un formalisme moderne, contre ses premières tentatives, se doit d'envisager une telle matérialité pour la littérature.

Jeune docteur en littérature et langue françaises de l'Université de Paris 3 Sorbonne-Nouvelle, Jérémy Naïm est spécialiste du XIX ${ }^{\mathrm{e}}$ siècle, de la littérature narrative, des rapports entre presse et littérature et des questions de théorie littéraire. Sa thèse portait sur le récit enchâssé au XIX ${ }^{\mathrm{e}}$ siècle, notion dont il critique la pertinence pour expliquer le faire d'œuvres très hétérogènes.

\section{Notes}

1 Roland Barthes, "Le retour du poéticien » [paru dans La Quinzaine littéraire, octobre 1972], Essais critiques IV. Le Bruissement de la langue, Paris, Seuil, coll. "Points », 1984, p. 201. Cité par Franc Schuerewegen, "Genette devient écrivain", Fabula-LbT, n 10, "L'aventure poétique », décembre 2012 (en ligne), URL : http://www.fabula.org/lht/10/schuerewegen.html, page consultée le 17 février 2017. 
2 Pour une histoire détaillée de l'approche sociologique, voir l'article récent de Pierre Popovic, «La sociocritique. Définition, histoire, concepts, voies d'avenir », Pratiques, 2011 (n 151-152), pp. 7-38.

3 Voir par exemple Alain Vaillant, "Pour une histoire de la communication littéraire », Revue d'histoire littéraire de la France 3/2003 (Vol. 103), pp. 549-562.

4 Marie-Ève Thérenty, «Pour une poétique historique du support», Romantisme 1/2009 (n 143), pp. 109-115.

${ }^{5}$ L'édition de 1883 est probablement fautive ici et écrit «le». La version publiée en journal met « de ».

${ }^{6}$ Maupassant, «La Bécasse », Contes de la bécasse, Paris, Rouveyre et Blond, 1883, pp. 10-12.

7 Sur ce point, voir Tzvetan Todorov, «Les hommes-récits » [1968], Poétique de la prose, Paris, Seuil, coll. «Poétique », 1971, p. 82.

8 Cité par Peter L. Caracciolo, The Arabian Nights in English Literature, London, St. Martin's Press, 1988, p. 1.

9 Emmanuelle Grandadam a montré l'importance de la composition dans les recueils publiés par Maupassant, notamment dans son ouvrage Contes et nouvelles de Maupassant : pour une poétique du recueil, Rouen, PURH, coll. « Maupassant », 2007.

10 Albert Delpit, «Une visite nocturne », Le Gaulois, seizième année, troisième série, no 135,28 novembre 1882, p. 1.

${ }^{11}$ Voir ses travaux sur La Littérature an quotidien, Paris, Seuil, coll. « Poétique », 2007.

12 Cette porosité entre nouvelle et chronique a amené Marie-Françoise Melmoux-Montaubin, dans le cadre de son travail d'édition des chroniques avec Ánne Geisler-Szmulewicz, a proposé la catégorie de «chronique-nouvelle». Voir son article "Maupassant chroniqueur: le cas des "chroniques-nouvelles" ", Bulletin Flaubert-Maupassant, 2015 (nº 31), p. 225-242.

13 Maupassant, «Histoire corse », Gil Blas, troisième année, numéro 744, $1^{\text {er }}$ décembre 1881, p. 1.

14 Maupassant, «Histoire d'un chien », Le Gaulois, treizième année, deuxième série, numéro 628, 2 juin 1881, p. 1.

15 Maupassant, « Magnétisme », Gil Blas, quatrième année, numéro 869, 5 avril 1882, p. 1.

16 Ferdinand Brunetière, "Les Nouvelles de M. de Maupassant », Revue des Deux Mondes, t. 89,1888 , pp. $703-704$.

17 Le retour de la première personne dans le roman du XXe siècle obéit à des enjeux complètement différents, malheureusement trop complexes pour être abordés ici. 


\section{Bibliographie}

\section{Sources}

Ferdinand Brunetière, "Les Nouvelles de M. de Maupassant», Revue des Deux Mondes, t. 89, 1888, p. 693-704.

Albert Delpit, "Une visite nocturne », Le Gaulois, seizième année, troisième série, n 135,28 novembre 1882, p. 1.

Guy de Maupassant, «Histoire corse », Gil Blas, troisième année, numéro 744, $1^{\text {er }}$ décembre 1881 , p. 1.

Guy de Maupassant, « Histoire d'un chien », Le Gaulois, treizième année, deuxième série, numéro 628, 2 juin 1881, p. 1.

Guy de Maupassant, "Magnétisme », Gil Blas, quatrième année, numéro 869, 5 avril 1882 , p. 1.

Guy de Maupassant, Contes de la bécasse, Paris, Rouveyre et Blond, 1883.

\section{Ouvrages et articles}

Roland Barthes, «Le retour du poéticien » [La Quinzaine littéraire, octobre 1972], Essais critiques IV. Le Bruissement de la langue, Paris, Seuil, coll. « Points », 1984.

Pierre Bourdieu, Les Règles de l'art. Genèse et structure du champ littéraire, Paris, Seuil, coll. «Libre examen », 1992.

Peter L. Caracciolo, The Arabian Nights in English Literature, London, St. Martin's Press, 1988.

Roger Chartier et Henri-Jean Martin (dir.), Histoire de l'édition française [1983-1986], Paris, Fayard et Cercle de la librairie, 1989-1991, 4 vol.

Roger Chartier et Guglielmo Cavallo (dir.), Histoire de la lecture dans le monde occidental [1995], Paris, Seuil, coll. « Points / Histoire », 2001.

Robert Escarpit, Sociologie de la littérature, Paris, Presses universitaires de France, coll. «Que sais-je? », 1958.

Gérard Genette, Seuils, Paris, Seuil, coll. Poétique, 1987.

Emmanuèle Grandadam, Contes et nouvelles de Maupassant: pour une poétique du recueil, Rouen, PURH, coll. «Maupassant », 2007. 
Marie-Françoise Melmoux-Montaubin, «Maupassant chroniqueur: le cas des "chroniques-nouvelles" ", Bulletin Flaubert-Maupassant, 2015 (n 31), pp. 225-242.

Jean-Yves Mollier (dir.), Le Commerce de la libraire en France au XIX siècle (1798-1914), Paris, IMEC, éd. De la Maison des sciences de l'homme, 1997.

Jean-Yves Mollier et Jacques Michon (dir.), Les Mutations du livre et de l'édition dans le monde du XVIII siècle à l'an 2000, actes du colloque international (Sherbrooke, 2000), Paris et Saint-Nicolas, L'Harmattan et Presses de l'Université de Laval, 2001.

Pierre Popovic, "La sociocritique. Définition, histoire, concepts, voies d'avenir », Pratiques 2011 (n 151-152), pp. 7-38.

Marie-Ève Thérenty, "Pour une poétique historique du support», Romantisme $1 / 2009$ (n 143), pp. 109-115.

Tzvetan Todorov, Poétique de la prose, Paris, Seuil, coll. « Poétique », 1971.

Alain Vaillant, "Pour une histoire de la communication littéraire ", Revue d'bistoire littéraire de la France 3/2003 (Vol. 103), pp. 549-562. 